\title{
Posterior walker assisted on gait performance in children with spastic diplegic cerebral palsy \\ ${ }^{1}$ Yu-Ju Chih, yuju519@gmail.com \\ ${ }^{2}$ Chung Huang Yu, chyu@ym.edu.tw \\ ${ }^{3} \mathrm{Li}-\mathrm{Ru}$ Chen, gracelr@ms1.mmh.org.tw \\ Institute of Rehabilitation Science and Technology, \\ National Yang-Ming University, Taiwan
}

\begin{abstract}
To make children who may lose some basic skills to accomplish walking or may have delayed in walking development is a very important issue. Gait in these children may exhibit the following characteristics: poor motor control, poor balance, muscle strength insufficiently, and muscle incoordination. Their mobility may depend upon the use of assistive devices such as canes, walkers, etc. These aids can improve balance and stability or provide body-weight support. They also facilitate forward progression of gait. However, selection and adjustment of walkers are often based on a subjective impression of the children's gait observed by the physician and therapists.

There seem not enough information regarding proper selection in choosing the walker design to best suit the children or in comparing the effects on gait load and stability of one design versus the others. Previous researches have studied children with CP and walker usage about lower extremity movement. However, these studies have not quantified upper extremity motions. From the viewpoint of biomechanics, the weight bearing of whole body and the force of upper extremity may influence the usage of walker. So, walker selection requires further study on the full range of upper and lower extremity.

The purpose of this study is to analyze the effects of different gait performances of spastic diplegic CP who use different heights of posterior walkers for ambulation.

Four spastic diplegic cerebral palsies, of average age 6.75 years, were enrolled in this study. They are all

familiarized themselves with posterior walkers. Gait characteristics were evaluated and observed by computer-based kinematics gait analysis using EVaRT 4.6 Motion Analysis, and force analysis by AMTI force plate and force transducers.

The conclusion of this study is the different heights of posterior walker would have different effect on gait performance while spastic diplegic cerebral palsy ambulated with walker. In clinic, we should adapt the walkers according to different goals and different abilities of users.

Keywords: walker, motion analysis, cerebral palsy, gait.

\section{Introduction}

Walking is an important basic skill for human. For the developing children, they can accept more stimuli from environment and other people by walking around. They also can development other skills more maturer and finish many functional activities by walking. Finally, they can be independent in their activities of daily life[1].

Walking is also an important index in childhood development. It means that children from quadruped crawling and biped kneeling to biped walking. It can increase muscle contraction and range of motion of lower extremities, and enhance trunk control[1, 2]. But some children can’t do walking well congenitally or acquired.
\end{abstract}


They may have disability in walking or delay in motor development. Cerebral palsy is a common group in clinic.

Cerebral palsy (CP) is a condition characterized by a motor disorder that is usually diagnosed during the early stages of life. It occurs from brains damage and has symptoms including poor motor control, postural instability, abnormal muscle tone and uncoordinated motor pattern. And it also has some problems in sensation, cognation, speech and psychosocial[3, 4]. Many CP children have difficulties in walking independently because of poor muscle strength, abnormal muscle tone, or poor postural control[5-8]. They often be prescribed with walkers to help stability and support when ambulation, especially spastic diplegic CP[9]. Their cerebral motor cortices have damage and their four limbs are all involved, but the legs more affected than the arms. They have some specific characteristics: increased muscle tone, abnormal motor pattern, joints contracture due to small range of motion, insufficient muscle strength of lower extremity and trunk. In ambulation, they may need some assistive devices to help them. Two types of walker have been advocated for CP in clinic. One is anterior walker, which is positioned in front of the user, the other is posterior walker, which is behind the child. The prescription of the walkers should be well-considered. The ability and limitation of users, the structures and parameters of the walkers, and the usage of the users to walkers are all should be considered. And it should be quantified and objective. However, it is still decided by subjective views of therapists and physician in clinic. So we can't understand how to match the users' goal with the walker parameters and the users' abilities efficiently and currently.

Many researchers have agreed that the posterior walker is better than anterior walker, in the light of upright postures, decreased double support time and increased velocity[10-14].

The purpose of this study is to analyze the gait performance of children with spastic diplegic cerebral palsy when these children use posterior walker with different heights.

\section{Methods}

\subsection{Subjects}

A convenience sample of four children (2 males, 2 females) with the diagnosis of spastic diplegic CP were studied. Their mean age was 6.75 years, mean height was $116.25 \mathrm{~cm}$, their mean height from greater trochanter to floor was $65.5 \mathrm{~cm}$, and their mean body weight was $21 \mathrm{~kg}$. Inclusion criteria was as follows:

(1) Spastic diplegic cerebral palsy.

(2) Use posterior walker as walking aid.

(3) Can understand verbal commands.

(4) Be able to ambulate with posterior walker at least 5 meters.

(5) No orthopedic surgery in 6 months.

Informed consent was obtains from the parents of all subjects before study.

\subsection{Experiment setup}

The posterior walker used in this study had been modified by adding two handle force transducers. The posterior walker had four wheels. The front wheels were limited anterior direction, the back could only roll forwards. The height of handle to the floor could be adjusted from $58 \mathrm{~cm}$ to $77 \mathrm{~cm}$.

The kinematics data of upper extremity and lower extremity was recorded using Evart 4.6 motion analysis system with a 10 cameras system.

\subsection{Procedures}

The posterior walker were adjusted in five different heights: the standard $(\mathrm{N})$ (the height of great trochanter is 
equal to the height of walker's handle), one inch higher (H1), two inches higher (H2), one inch lower (L1), and two inches lower (L2). The subject would wear underclothes and couldn't have any object that would reflect infrared in their bodies. There was a 7-meters walkway in this study. Before testing, the subjects would have opportunities to practice walking and the staff would calibrate the hardware and the environment. The subjects ought to have at least three effective trails for every height, once with each height in random order. They walked at a self-selected comfortable speed. They would have rested for 10 minutes after every height. The markers would be stuck on the subjects' bodies by the same staff for higher reliability. The reflective markers on the subjects were attached to the following anatomic locations: head markers at top, lateral, front and back, bilateral shoulder markers at the acromion process, bilateral elbow markers at the olecranon, bilateral wrist markers at the ulnar styloid, sacral marker at the midpoint on the line between both posterior superior iliac spines, bilateral greater trochanter markers at the lateral greater trochanter, bilateral pelvic markers at both anterior superior iliac spines, bilateral thigh markers at the midpoint on the line between the great trochanter and the center of the knee, bilateral knee markers at the lateral knee joint, bilateral shank markers at the midpoint on the line between the lateral knee joint and the later malleolus, bilateral ankle markers at lateral malleolus, bilateral heel markers at , and bilateral forefoot markers at the $2^{\text {nd }}$ metatarsal head.

\subsection{Data analysis}

There are high heterogeneities between different CP subjects. So, this study would compare the effect in different heights of the same subject.

The variables of velocity, cadence, and step length were calculated:

(1). Velocity: calculated by subtracting the X coordinate of the sacral marker at the end of a gait cycle from its $\mathrm{X}$ coordinate at the beginning of a gait cycle and then dividing the times of this cycle.

(2). Cadence: calculated by subtracting the times of the end of a gait cycle from its beginning of a gait cycle and then dividing the steps of this gait cycle.

(3). Step length: the distance was from one foot-floor contact to the foot-floor contact of opposite leg. It calculated by subtracting the $\mathrm{X}$ coordinate of the second foot-floor contact from the first one.

\section{Results}

\section{Temporo-spatial variable on gait performance}

The results are showed in

Table 1 Table 3

Subject A and D had poor motor control in walking with walker., and subject B and C had good motor control in walking with walker. On gait performance, we could find that the better performances of all subjects were the same.

Subject A: had the faster average velocity, longest step length and highest cadence in L1 walker.

Subject B: had the faster average velocity, longest step length and highest cadence in H1 walker.

Subject C: had the faster average velocity, longest step length and highest cadence in $\mathrm{N}$ walker.

Subject D: had the faster average velocity, longest step length and highest cadence in H2 walker. 
Table 1 Average velocity

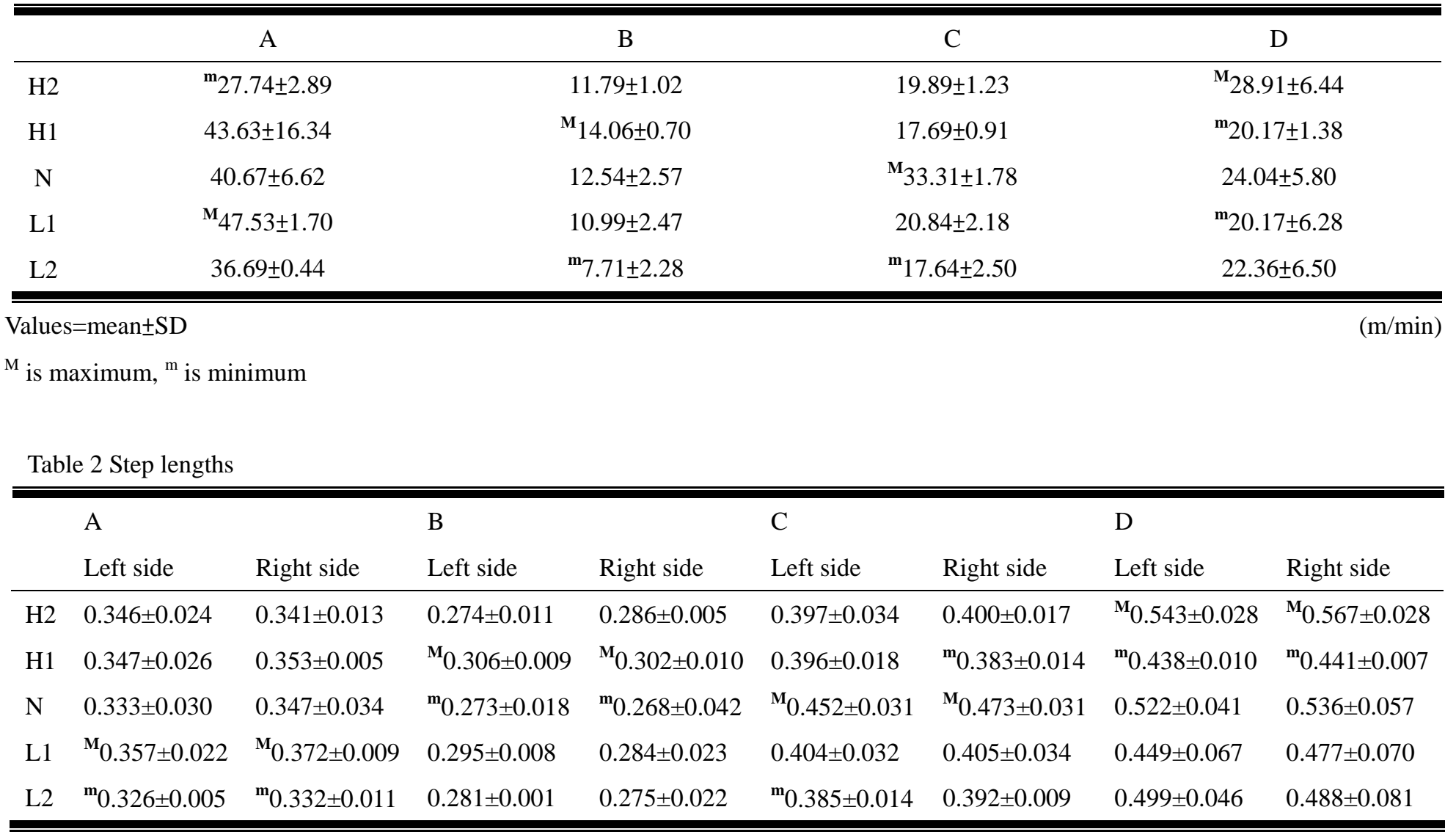

Values $=$ mean \pm SD

${ }^{\mathrm{M}}$ is maximum, ${ }^{\mathrm{m}}$ is minimum

Table 3 Cadence

\begin{tabular}{ccccc}
\hline \hline & $\mathrm{A}$ & $\mathrm{B}$ & $\mathrm{C}$ & $\mathrm{D}$ \\
\hline $\mathrm{H} 2$ & $\mathbf{m}_{40.74 \pm 5.04}$ & $27.80 \pm 3.91$ & $41.45 \pm 1.58$ & $\mathbf{M}_{38.06 \pm 6.55}$ \\
$\mathrm{H} 1$ & $59.92 \pm 4.65$ & $\mathbf{M}_{41.09 \pm 0.27}$ & $37.93 \pm 3.43$ & $36.81 \pm 2.26$ \\
$\mathrm{~N}$ & $58.41 \pm 4.56$ & $33.82 \pm 1.79$ & $\mathbf{m}_{58.19 \pm 2.00}$ & $37.05 \pm 5.30$ \\
$\mathrm{~L} 1$ & $\mathbf{M}_{63.88 \pm 4.54}$ & $39.01 \pm 0.66$ & $40.02 \pm 4.71$ & $34.60 \pm 6.77$ \\
$\mathrm{~L} 2$ & $54.84 \pm 1.57$ & $\mathbf{m}_{19.75 \pm 3.26}$ & $\mathbf{m}_{37.64 \pm 5.45}$ & $\mathbf{m}_{34.03 \pm 4.36}$ \\
\hline \hline
\end{tabular}

Values $=$ mean $\pm S D$

(steps/min)

${ }^{\mathrm{M}}$ is maximum, ${ }^{\mathrm{m}}$ is minimum 


\section{Discussion}

The height of the walker was one of the convenient way to adjust the walker for different needs or goals and different abilities of users. From the results of this study, we could find that the different heights have the different effects for individuals, although the regularizations between subjects were unobviously, but we could find the suitable height for the subject in different goals.

The samples in this study were a little small, and the subjects' abilities were too divers. The grading of subject's criteria wasn't detail enough, so the results of this study couldn't apply to the subjects of spastic diplegic cerebral palsy.

On gait performance, if the functional performances were our goals, the users would have faster walking velocity, longer step lengths and higher cadence that could let them walk smooth and steady. In our study, we could find that the standard height that used in clinic wasn't the most suitable height for our subjects.

Future works can have more subjects, and the ability of the subject can be more similar and detail to have agreeable results.

\section{Acknowledgement}

This study was aided by department of rehabilitation of Mackay Memorial Hospital.

\section{References}

1. Bax, M., walking. Developmental Medicine \& Child Neurology, 1991. 33(6): p. 471-2.

2. Rose, J. and J.G. Gamble, Human walking. 2nd ed. Ch.1 Human Locomotion, ed. H.J.R. Veene T. Inman, and Frank Todd. 1994, Baltimore: Williams and Wilkins.

3. Gage, J.R., Gait analysis in cerebral palsy. Ch.1 Introduction. 1991, London: Mac Keity Press.
4. Levitt, S., Treatment of cerebral palsy and motor delay. 4th ed. Ch.1 The clinical picture for therapy and management. 2004, Oxford [England]; Cambridge, Mass., USA: Blackwell Publishing.

5. Scholtes, V.A., et al., Clinical assessment of spasticity in children with cerebral palsy: $a$ critical review of available instruments. Dev Med Child Neurol, 2006. 48(1): p. 64-73.

6. Leonard, C.T., H. Hirschfeld, and H. Forssberg, The development of independent walking in children with cerebral palsy. Dev Med Child Neurol, 1991. 33(7): p. 567-77.

7. Bottos, M. and C. Gericke, Ambulatory capacity in cerebral palsy: prognostic criteria and consequences for intervention. Dev Med Child Neurol, 2003. 45(11): p. 786-90.

8. Stanger, M. and S. Oresic, Rehabilitation approaches for children with cerebral palsy: overview. J Child Neurol, 2003. 18 Suppl 1: p. S79-88.

9. Sussman, M.D. and M.D. Aiona, Treatment of spastic diplegia in patients with cerebral palsy. $\mathrm{J}$ Pediatr Orthop B, 2004. 13(2): p. S1-12.

10. Sutherland, D.H., et al., The development of mature gait. J Bone Joint Surg Am, 1980. 62(3): p. 336-53.

11. Rose, J., J.M. Medeiros, and R. Parker, Energy cost index as an estimate of energy expenditure of cerebral-palsied children during assisted ambulation. Developmental Medicine \& Child Neurology, 1985. 27(4): p. 485-90.

12. Levangie, P., Chimera M, Johnson M, Robinson F, Wobeskya L., The effects of posterior rolling walkers vs. the standard rolling walker on gait characteristics of children with spastic cerebral palsy. Phys Occup Ther Pediatr, 1989: p. 1-17.

13. Logan, L., C.D. Ciccone, and K. Byers-Hinkley, 
Anterior versus posterior walkers: a gait analysis study. Developmental Medicine \& Child Neurology, 1990. 32(12): p. 1044-8.

14. Greiner, B.M., J.M. Czerniecki, and J.C. Deitz, Gait parameters of children with spastic diplegia: a comparison of effects of posterior and anterior walkers. Archives of Physical Medicine \& Rehabilitation, 1993. 74(4): p. 381-5. 